\title{
Effects of Nonlinear Elasticity and Electromechanical Coupling on Optical Properties of InGaN/GaN and AlGaN/AlN Quantum Wells
}

\author{
S.P. LEPKOWSKI ${ }^{a, *}$ AND J.A. MAJEWSKI ${ }^{b}$ \\ ${ }^{a}$ Unipress, Institute of High Pressure Physics, Polish Academy of Sciences \\ Sokołowska 29, 01-142 Warszawa, Poland \\ ${ }^{b}$ Institute of Theoretical Physics and Interdisciplinary Center for Materials \\ Modeling, Warsaw University, Hoża 69, 00-681 Warszawa, Poland

\begin{abstract}
We present theoretical studies of effects of the nonlinear elasticity and the electromechanical coupling on the optical properties of InGaN/GaN and $\mathrm{AlGaN} / \mathrm{AlN}$ quantum wells. In these structures, due to the lattice misfit between constituents, the quantum wells are compressively strained and the intrinsic hydrostatic pressure is present. Therefore, the nonlinear elasticity is investigated by taking into account the pressure dependence of elastic stiffness tensor for the strained quantum wells. We show that this effect leads to (i) decrease in the volumetric strain and (ii) increase in the polarization-induced built-in electric field in the quantum wells. Consequently, the interband transition energies in the quantum wells decrease when the nonlinear elasticity of nitrides is considered. On the other hand, we show that the effect of electromechanical coupling, i.e., co-existence of ordinary and converse piezoelectric effects results in increase in the interband transition energies in the considered quantum wells. It turns out that the influence of the nonlinear elasticity on the optical properties is stronger than the influence of electromechanical coupling for InGaN/GaN quantum wells, while for AlGaN/GaN the opposite situation is observed.
\end{abstract}

PACS numbers: 78.67.De, 62.20.Dc, 77.65.-j

\section{Introduction}

Wurtzite group-III nitride quantum wells (QWs) have been recently extensively studied. The optical properties of these structures depend crucially on the

*corresponding author; e-mail: slawek@unipress.waw.pl 
strain and the built-in piezoelectric field, which via quantum confined Stark effect influence energy and probability of the interband transitions [1]. A simple approach based on linear dependences of the piezoelectric polarization and stress on strain, i.e. on linear, ordinary piezoelectric effect and linear elastic theory, has been conventionally used to determine the strain and the built-in electric field nitride quantum structures [1-3]. Recently, higher order electromechanical effects have been also taken into account. Firstly, it has been found that the spontaneous and piezoelectric polarizations for nitrides show strong nonlinear dependence of strain [4]. Secondly, the influence of the converse piezoelectric effect, i.e. dependence of the stress on the built-in electric field, has been studied in nitride quantum wells and quantum dots [5-7]. Thirdly, the importance of nonlinear elasticity in nitride heterostructures has been demonstrated, showing that the elastic constant depend significantly on stress, particularly on the hydrostatic pressure [8, 9].

In this paper, we investigate the effects of the nonlinear elasticity and the electromechanical coupling on the optical properties of InGaN/GaN and AlGaN/AlN QWs. In these structures, due to the lattice misfit between constituents, the QWs are compressively strained and the intrinsic hydrostatic pressure is present. Therefore, the pressure dependence of elastic constants is expected to be important for determination of the strain and the built-in electric field. Here, we study also the effect of electromechanical coupling, i.e., self-consistent treatment of the ordinary and converse piezoelectric effects in the considered QWs [7]. Interestingly, these two phenomena influence the energies of the interband transitions in opposite way, but usually one effect dominates over the other.

\section{Nonlinear elasticity in nitride QWs}

The effect of nonlinear elasticity in nitrides has been extensively investigated by us in Ref. [9], where we have calculated pressure dependencies of elastic constants for GaN, InN and AlN in zinc-blende and wurtzite crystallographic phases. As a kind of summary, we present the pressure dependence of elastic constants in Table. In this section, we want to examine the effect of nonlinear elasticity on optical properties of nitride QWs. We consider two types of coherently grown heterostructures, InGaN/GaN QWs on GaN and AlGaN/AlN QWs on AlN. In these structures, due to the lattice misfit between constituents, the QWs are compressively strained and the intrinsic hydrostatic pressure is present.

Let us start the theoretical analysis by considering the Hook law, for a compressively strained QW grown in the polar [0001] direction of wurtzite crystal. It takes the following form:

$$
\left[\begin{array}{c}
\sigma_{x x} \\
\sigma_{y y} \\
\sigma_{z z}
\end{array}\right]=\left[\begin{array}{c}
F \\
F \\
0
\end{array}\right]=\left[\begin{array}{lll}
C_{11}(P) & C_{12}(P) & C_{13}(P) \\
C_{12}(P) & C_{11}(P) & C_{13}(P) \\
C_{13}(P) & C_{13}(P) & C_{33}(P)
\end{array}\right]\left[\begin{array}{c}
\varepsilon_{x x} \\
\varepsilon_{y y} \\
\varepsilon_{z z}
\end{array}\right],
$$

where $F$ denotes the biaxial stress due to the lattice misfit between QW and bar- 
TABLE

Pressure dependent elastic constants, $C_{\alpha \beta}(P)$, for wurtzite nitrides obtained using $a b$ initio calculations. Data are taken from Ref. [9].

\begin{tabular}{c|c|c|c}
\hline \hline & GaN & InN & AlN \\
\hline$C_{11}$ & $366+4.88 P-0.038 P^{2}$ & $229+3.66 P-0.012 P^{2}$ & $397+3.78 P-0.009 P^{2}$ \\
$C_{12}$ & $139+3.69 P-0.015 P^{2}$ & $120+3.51 P+0.002 P^{2}$ & $143+2.78 P+0.004 P^{2}$ \\
$C_{13}$ & $98+3.75 P-0.029 P^{2}$ & $95+4.11 P-0.014 P^{2}$ & $112+3.34 P-0.016 P^{2}$ \\
$C_{33}$ & $403+6.54 P-0.079 P^{2}$ & $234+4.26 P-0.049 P^{2}$ & $372+3.65 P-0.066 P^{2}$ \\
$C_{44}$ & $97+0.49 P-0.007 P^{2}$ & $49+0.15 P-0.004 P^{2}$ & $116+0.75 P-0.0008 P^{2}$
\end{tabular}

riers [9]. The off-diagonal components of the strain tensor, $\varepsilon_{\lambda \delta}$, and the stress tensor, $\sigma_{\lambda \delta}$, are zeros, due to symmetry conditions and have been omitted. From Eq. (1), it is easily seen that $\varepsilon_{x x}=\varepsilon_{y y}$ and

$$
\varepsilon_{z z}=-\frac{2 C_{13}(P)}{C_{33}(P)} \varepsilon_{x x} .
$$

The in-plane strain component is given by

$$
\varepsilon_{x x}=\frac{a_{\mathrm{br}}-a_{\mathrm{qw}}}{a_{q w}},
$$

where $a_{\mathrm{qw}}$ and $a_{\mathrm{br}}$ are the lateral lattice constants of QWs and barriers. For simplicity, we have assumed that barriers are relaxed and much thicker than the QWs. Now, let us introduce the usual definition of hydrostatic pressure, $P=-\frac{1}{3} \operatorname{Tr}\left(\sigma_{\alpha \beta}\right)$, which in our case leads to the expression

$$
P=-\frac{2}{3} F=-\frac{2}{3}\left\{C_{11}(P)+C_{12}(P)-2 \frac{\left[C_{13}(P)\right]^{2}}{C_{33}(P)}\right\} \varepsilon_{x x} .
$$

To determine the built-in electric field in the QWs, we consider first spontaneous and piezoelectric polarization. The non-vanishing $z$-components (in the [0001] direction, along $c$-axis) of the total piezoelectric polarization in the QW and barriers are given by

$$
P_{\mathrm{tot}, i}=2 e_{31, i} \varepsilon_{x x, i}+e_{33, i} \varepsilon_{z z, i}+P_{\mathrm{spon}, i}, \quad i=\mathrm{qw}, \mathrm{br},
$$

where $e_{31, i}$ and $e_{33, i}$ are elements of the piezoelectric tensor and $P_{\mathrm{spon}, i}$ is the spontaneous polarization [1]. In accordance with the assumption that the barriers are much thicker than the QWs, the $z$-component of the built-in electric field vector in the $\mathrm{QW}$ is given by

$$
E_{\mathrm{qw}}=\frac{P_{\mathrm{tot}, \mathrm{br}}-P_{\mathrm{tot}, \mathrm{qw}}}{\chi_{\mathrm{qw}}}
$$

where $\chi_{\text {qw }}$ is the static dielectric constant for the QWs [10]. Then, using Eqs. (2) and (5), and additionally assuming that the barriers are unstrained, i.e., $P_{\text {tot }, \mathrm{br}}=P_{\mathrm{spon}, \mathrm{br}}$, one gets

$$
E_{\mathrm{qw}}=\frac{1}{\chi_{\mathrm{qw}}}\left[P_{\mathrm{spon}, \mathrm{br}}-P_{\mathrm{spon}, \mathrm{qw}}-2\left(e_{31, \mathrm{qw}}-\frac{C_{13}(P)}{C_{33}(P)} e_{33, \mathrm{qw}}\right) \varepsilon_{x x}\right] .
$$




\section{Electromechanical coupling in nitride QWs}

In order to investigate the effect of electromechanical coupling in nitride QWs (i.e., ordinary and converse piezoelectric effects), let us express the diagonal elements of the stress tensor in wurtzite [0001] QW, as a function of strain and the built-in electric field [7]:

$$
\left[\begin{array}{c}
\sigma_{x x} \\
\sigma_{y y} \\
\sigma_{z z}
\end{array}\right]=\left[\begin{array}{c}
F \\
F \\
0
\end{array}\right]=\left[\begin{array}{lll}
C_{11} & C_{12} & C_{13} \\
C_{12} & C_{11} & C_{13} \\
C_{13} & C_{13} & C_{33}
\end{array}\right]\left[\begin{array}{c}
\varepsilon_{x x} \\
\varepsilon_{y y} \\
\varepsilon_{z z}
\end{array}\right]+\left[\begin{array}{c}
e_{31} \\
e_{31} \\
e_{33}
\end{array}\right] E_{\mathrm{qw}} .
$$

From Eq. (8), one gets again the equality $\varepsilon_{x x}=\varepsilon_{y y}$ and

$$
\varepsilon_{z z}=-\frac{2 C_{13}}{C_{33}} \varepsilon_{x x}+\frac{e_{33}}{C_{33}} E_{\mathrm{qw}} .
$$

The in-plane strain in the QW is of course expressed by Eq. (3). According to Eqs. (5) and (6), the built-in electric field in the QW with unstrained and wide barriers is given by

$$
E_{\mathrm{qw}}=\frac{1}{\chi_{\mathrm{qw}}}\left[P_{\mathrm{spon}, \mathrm{br}}-P_{\mathrm{spon}, \mathrm{qw}}-2 e_{31, \mathrm{qw}} \varepsilon_{x x}-e_{33, \mathrm{qw}} \varepsilon_{z z}\right]
$$

Substituting Eq. (9) into Eq. (10) and solving for $E_{\mathrm{qw}}$ yields

$$
E_{\mathrm{qw}}=\frac{1}{\chi_{\mathrm{qw}}+\frac{\left(e_{33, \mathrm{qw}}\right)^{2}}{C_{33}}}\left[P_{\mathrm{spon}, \mathrm{br}}-P_{\mathrm{spon}, \mathrm{qw}}-2\left(e_{31, \mathrm{qw}}-\frac{C_{13}}{C_{33}} e_{33, \mathrm{qw}}\right) \varepsilon_{x x}\right] \text {. }
$$

Equations (3), (9) and (11) determine the strain and the built-in electric field in the QW with the electromechanical coupling effect taken into account.

\section{Results and discussion}

In order to study the influence of the nonlinear elasticity and electromechanical coupling effects on interband transition energies, $E_{\mathrm{c}-\mathrm{v}}$, in nitride QWs one has to solve the Schrödinger equation for electronic states in the heterostructure. For this purpose, we use the $\boldsymbol{k} \cdot \boldsymbol{p}$ method with the strain and the built-in electric field included in the $\boldsymbol{k} \cdot \boldsymbol{p}$ Hamiltonian in a standard manner (see e.g. Ref. [2] for details). The conduction and valence bands are treated separately, since the energy gap of quantum well material is sufficiently large in both AlGaN/AlN and InGaN/GaN QWs. Additionally, we are interested only in the maximum of the valence band energies and thus the $6 \times 6 \boldsymbol{k} \cdot \boldsymbol{p}$ system of differential equations for six component spinor of envelope functions reduces to a single equation for the heavy-hole component [2]. All material parameters used in the calculations are listed in our earlier paper [9]. In this place, we would like to notice that strain dependent piezoelectric constants of nitrides have been taken into account.

In Fig. 1 we present differences in $E_{\mathrm{c}-\mathrm{v}}$, obtained by taking into account the nonlinear elasticity (squares) and electromechanical coupling (circles) effects for InGaN/GaN QWs with In content varying from 0.1 to 0.3 . One can see that both effects contribute to the values of $E_{\mathrm{c}-\mathrm{v}}$ in opposite way. The nonlinear elasticity 


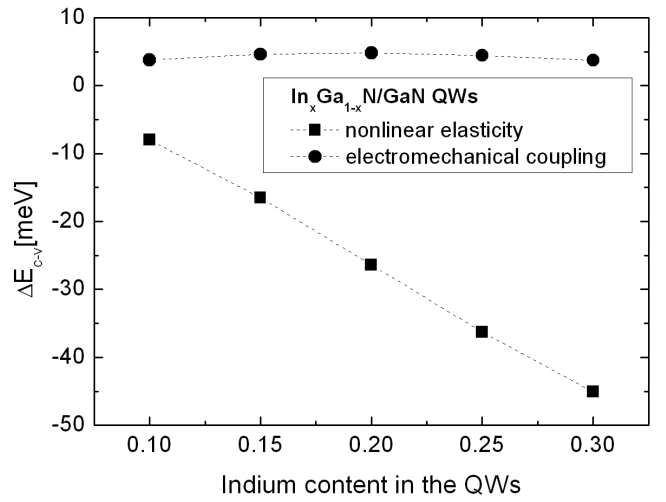

Fig. 1. The differences in the interband transition energies for InGaN/GaN QWs (with In content varying from 0.1 to 0.3 ) obtained by taking into account the nonlinear elasticity (squares) and electromechanical coupling (circles) effects. Dashed lines are added to guide the eye.

effect leads to decrease in $E_{\mathrm{c}-\mathrm{v}}$, while the electromechanical coupling increases $E_{\mathrm{c}-\mathrm{v}}$. In InGaN/GaN QWs, the effect of nonlinear elasticity is much stronger than the electromechanical coupling.

In Fig. 2 we show differences in $E_{\mathrm{c}-\mathrm{v}}$, due the nonlinear elasticity (squares) and electromechanical coupling (circles) effects for AlGaN/AlN QWs with Al content varying from 0.9 to 0.5 . Again, one can see that the nonlinear elasticity leads to decrease in $E_{\mathrm{c}-\mathrm{v}}$, while the electromechanical coupling increases $E_{\mathrm{c}-\mathrm{v}}$. However, this time the contribution to $E_{\mathrm{c}-\mathrm{v}}$ from the electromechanical coupling is larger than the contribution from the nonlinear elasticity.

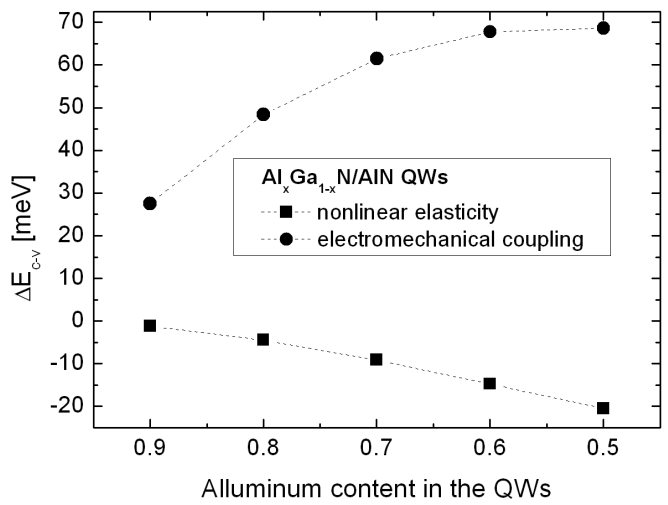

Fig. 2. The differences in the interband transition energies for AlGaN/AlN QWs (with $\mathrm{Al}$ content varying from 0.9 to 0.5 ) obtained by taking into account the nonlinear elasticity (squares) and electromechanical coupling (circles) effects. Data are presented in the direction of decreasing $\mathrm{Al}$ content in the QWs (and increasing the height of the barriers) to facilitate the comparison with Fig. 1. Dashed lines are added to guide the eye. 


\section{Conclusions}

In summary, we have studied the influence of the nonlinear elasticity and the electromechanical coupling effects on energies of the interband transitions in InGaN/GaN and AlGaN/AlN QWs. Our studies reveal that both effects contribute to the values of $E_{\mathrm{c}-\mathrm{v}}$ in opposite way. The nonlinear elasticity leads to decrease in $E_{\mathrm{c}-\mathrm{v}}$, while the electromechanical coupling increases $E_{\mathrm{c}-\mathrm{v}}$. In InGaN/GaN QWs, the effect of nonlinear elasticity is much stronger than the electromechanical coupling. For AlGaN/AlN QWs, the contribution to $E_{\mathrm{c}-\mathrm{v}}$ from the electromechanical coupling is larger than the contribution from the nonlinear elasticity. Obviously, for accurate determination of the interband transitions in these structures both effects should be taken into account.

\section{Acknowledgments}

This work was supported by the State Committee for Scientific Research, projects No. 4T11F00825 and 1P03B03729. Additionally, we acknowledge the use of computing facilities at ICM UW.

\section{References}

[1] V. Fiorentini, F. Bernardini, F. Della Sala, A. Di Carlo, P. Lugli, Phys. Rev. B 60, 8849 (1999).

[2] S.-H. Park, S.-L. Chuang, J. Appl. Phys. 87, 353 (2000).

[3] J.L. Sánchez-Rojas, J.A. Garrido, E. Muñoz, Phys. Rev. B 61, 2773 (2000).

[4] F. Bernardini, V. Fiorentini, Phys. Rev. B 64, 085207 (2001).

[5] E. Pan, J. Appl. Phys. 91, 3785 (2002).

[6] B. Jogai, J.D. Albrecht, E. Pan, J. Appl. Phys. 94, 6566 (2003).

[7] B. Jogai, J.D. Albrecht, E. Pan, Semicond. Sci. Technol. 19, 733 (2004).

[8] S.P. Łepkowski, J.A. Majewski, Solid State Commun. 131, 763 (2004).

[9] S.P. Łepkowski, J.A. Majewski, G. Jurczak, Phys. Rev. B 72, 245201 (2005).

[10] J.A. Majewski, G. Zandler, P. Vogl, J. Phys., Condens. Matter 14, 3511 (2002). 